\title{
Torsiflex: an automatic generator of torsional conformers. Application to the twenty proteinogenic amino acids
}

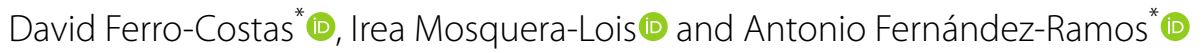

\begin{abstract}
In this work, we introduce TorsiFlex, a user-friendly software written in Python 3 and designed to find all the torsional conformers of flexible acyclic molecules in an automatic fashion. For the mapping of the torsional potential energy surface, the algorithm implemented in TorsiF lex combines two searching strategies: preconditioned and stochastic. The former is a type of systematic search based on chemical knowledge and should be carried out before the stochastic (random) search. The algorithm applies several validation tests to accelerate the exploration of the torsional space. For instance, the optimized structures are stored and this information is used to prevent revisiting these points and their surroundings in future iterations. TorsiFlex operates with a dual-level strategy by which the initial search is carried out at an inexpensive electronic structure level of theory and the located conformers are reoptimized at a higher level. Additionally, the program takes advantage of conformational enantiomerism, when possible. As a case study, and in order to exemplify the effectiveness and capabilities of this program, we have employed TorsiFlex to locate the conformers of the twenty proteinogenic amino acids in their neutral canonical form. TorsiFlex has produced a number of conformers that roughly doubles the amount of the most complete work to date.
\end{abstract}

Keywords: Conformations, Flexible molecules, Torsions, Preconditioned search, Stochastic search, Validation tests, Amino acids

\section{Graphical Abstract}
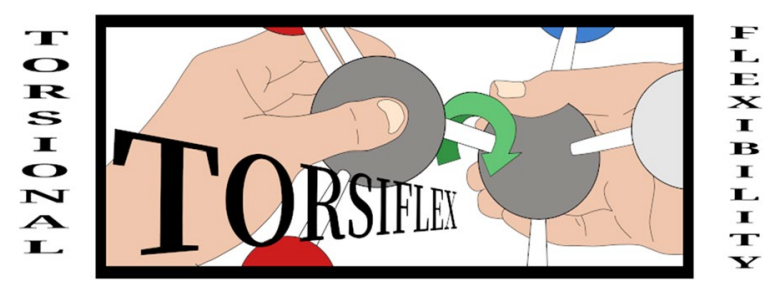

*Correspondence: david.ferro@usc.es; qf.ramos@usc.es

Centro Singular de Investigación en Química Biolóxica e Materiais Moleculares (CIQUS), Universidade de Santiago de Compostela,

15782 Santiago de Compostela, Spain

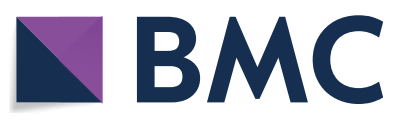

(c) The Author(s) 2021. Open Access This article is licensed under a Creative Commons Attribution 4.0 International License, which permits use, sharing, adaptation, distribution and reproduction in any medium or format, as long as you give appropriate credit to the original author(s) and the source, provide a link to the Creative Commons licence, and indicate if changes were made. The images or other third party material in this article are included in the article's Creative Commons licence, unless indicated otherwise in a credit line to the material. If material is not included in the article's Creative Commons licence and your intended use is not permitted by statutory regulation or exceeds the permitted use, you will need to obtain permission directly from the copyright holder. To view a copy of this licence, visit http://creativecommons.org/licenses/by/4.0/. The Creative Commons Public Domain Dedication waiver (http://creativeco mmons.org/publicdomain/zero/1.0/) applies to the data made available in this article, unless otherwise stated in a credit line to the data. 


\section{Introduction}

Flexible molecules are prone to adopt different geometries due to the internal rotation about single bonds. Each of these equilibrium structures is represented by a unique spatial configuration, that is, a conformational isomer. Temperature and relative stability decide the conformations that possess a significant population; thus, in astrochemical studies (low temperature), it suffices to identify the most stable conformers, whereas in combustion studies (high temperature) most or all conformers need to be characterized. However, even if we just focus on the most stable equilibrium structures, it is very complex to know a priori their location, and the computational effort is, in many situations, similar to the exploration of the whole configurational space. Therefore, the search and location of equilibrium structures in flexible molecules with multiple internal rotations demands an efficient sampling of the torsional potential energy surface (PES).

Some of the first and still valid ideas are based on the Metropolis Monte Carlo sampling [1-3], the eigenvectorfollowing algorithm [4-6], or the systematic search [7], although very recently machine learning [8-10], genetic [11], and meta-dynamics algorithms [12] are probing new routes. The application of any of these algorithms to large systems (cyclic or acyclic) usually requires improving their efficiency, and sometimes they are combined with knowledge-based methods that profit from databanks and/or diverse force field implementations but, at the same time, also rely heavily on them [13-19]. For systems of more modest size and for the location of the lowenergy conformers, it is possible to adopt semiempirical tight-binding [20], or a combination of low-level quantum mechanics $(\mathrm{QM})$ and molecular mechanics methods (MM) [21].

Torsiflex is a Python 3 code designed in the line of methods that handle flexible acyclic molecules of modest size (for instance, compounds of astronomical interest, amino acids and small peptides, organic molecules of a few tenths of atoms, etc.), with the objective of locating all the equilibrium structures of the torsional PES. The algorithm of Torsiflex combines preconditioned and stochastic approaches for the sampling of configurational space [22, 23]. By preconditioned search, we mean a combination of systematic search and chemistry-based knowledge. It has been reported that accounting just for the anticipated dispositions may miss relevant parts of the conformational space (an example is the $3^{N}$ estimator for $s p^{3}$ carbon atoms) [24]. For this reason, the preconditioned search followed by the stochastic exploration allows locating, not just the expected conformers, but also conformers that go beyond the chemically intuitive arrangements. As many other methods, our approach is complemented by dual-level (or two-level) calculations such that the conformational search can be carried out at an inexpensive level of calculation (low level, LL) whereas the final conformer refinement relies on more accurate calculations (high level, HL).

Most algorithms (ours included) entrust sampling strategies to LL (usually MM) with the idea of a fast exploration of the PES. This initial step is key in the location of the stationary points because these LL minima are adopted as the starting point for HL calculations. The main drawback of this procedure is that the LL and HL PESs should bear a certain degree of resemblance to grant that the most relevant LL regions are also of relevance at $\mathrm{HL}$; otherwise there is a risk of overlooking some of the low-energy equilibrium conformations. For this reason, we prefer to employ LL ab initio methods instead of semiempirical or MM methods, although the latter are substantially faster.

Independently of the subsequent handling of the LL information, the generation of a suitable set of LL structures is crucial at any rate, although the demand for HL information can be adjusted. Thus, we may be concerned only about the identification of the most stable conformer(s) among the bevy of them, as for instance when dealing with astrochemical studies, [25] or when interpreting high-resolution microwave spectra at cryogenic temperatures. [26] In other situations, as for instance when studying the mechanism of bioalcohols or other combustion biofuels, most of the HL conformers, if not all, need to be identified. [27] The latter is required to calculate thermodynamic properties from the gathered structural information. Particularly, these macroscopic properties can be extracted from a single generating function, that is, the partition function. There exist different strategies to incorporate the effect of several conformers in the partition function, such as the multi-structural harmonic-oscillator approximation (MS-HO) [27-29], the extended two-dimensional torsional method (E2DT) [30-32], the coupled torsional anharmonic approximation (MS-T) [33, 34], or composite methods $[35,36]$ among others. However, all of them share a common starting point toward the evaluation of more accurate partition functions: the search and location of the torsional conformers.

In this work, we aim to find all the conformations of the twenty proteinogenic amino acids, pAAs, in their neutral canonical form. From a biological point of view, they constitute the building blocks of proteins and are associated with the machinery of life [37]. From a chemical point of view, pAAs display remarkable conformational flexibility, a situation that is hampered by the presence of hydrogen bond interactions. These properties are crucial when studying the dynamics of the protein backbones. The number of torsions 
in amino acids, and consequently the number of conformers, varies greatly from one system to another. For example, glycine (Gly), the simplest amino acid, contains a total of three torsions, whereas other amino acids, like aspartic acid (Asp), glutamine (Gln) and methionine (Met), comprise up to 6 torsions. The most complicated situation can be encountered in arginine (Arg), whose conformational flexibility is determined by a total of 9 torsions. These aspects, together with their moderate molecular size, render pAAs as attractive targets to test Torsiflex.

From the experimental point of view, the studies by modern techniques as the laser ablation molecular-beam Fourier transform microwave (LA-MB-FTMW) carried out at cryogenic temperatures are contributing to reveal the intrinsic properties of pAAs in the absence of an interacting environment [38-47].

From a theoretical point of view, conformational studies on individual pAAs are extensive in literature, especially in the gas phase where their canonical (neutral uncharged) form prevails [48-72]. There are also studies that considered amino acids in their zwitterionic, cationic or anionic forms (the prevailing forms in aqueous solution). For example, Turan and Selçuki [69] studied glutamic acid (Glu) at the B3LYP/cc-pVTZ level and reported a total of 165 conformers for its canonical form, 63 for the zwitterionic forms, 199 for the anionic forms, 24 for the dianionic and 135 for the cationic form. However, the study of individual compounds prevents a fair comparison between amino acids due to the fact that each of them was analyzed at different electronic structure levels and by different searching algorithms.

Theoretical works that considered the twenty pAAs are scarce; Jamróz et al. [70] reported a total of 1684 canonical conformers on their study about chirality measures of $\alpha$-amino acids. More recently, Ropo et al. [71, 72] studied all proteinogenic amino acids at the PBE generalizedgradient exchange-correlation functional [73] with tier2 basis set and with corrections for van der Waals interactions. Notably, their search was not restricted to the arrangements arising from chemically intuitive dihedral angles, a common practice in conformational studies. Their dataset contains conformers for the twenty amino acids in their uncharged and zwitterionic forms, in addition to including dipeptides and the interaction with several divalent cations. Regarding the canonical forms, their database for the 20 pAAs contains a total of 3315 geometries, which is twice the number of conformers reported by Jamróz et al. a clear indication of the conformational richness accessible to the pAAs in the gaseous phase. In order to exemplify the effectiveness and capabilities of Torsiflex, we have searched for all conformers of the
20 pAAs in their canonical form and the results are compared with these previous works.

The manuscript is organized as follows: "Computational details" Section briefly describes the technicalities associated with the electronic structure calculations, "Algorithm and implementation" Section introduces Torsiflex and analyzes the algorithm implemented in it, "Results and discussion" Section presents the results for the pAAs establishing a comparison with previous studies, and "Conclusions" Section summarizes the main findings of this work.

\section{Computational details}

Torsiflex is a program written in Python 3 and includes a completely automated interface with Gaussian (versions 09 [74] and 16 [75]) and the two programs were employed for the location of the conformers of the twenty pAAs. The LL calculations involve geometry optimizations and Hessian calculations at the optimized geometries to ensure that the converged structure corresponds to an equilibrium geometry. TorsiFlex can function with any of the MM, semiempirical, Density Functional Theory (DFT), or $a b$ initio methods implemented in Gaussian. Our recommendation is to employ the split-valence 3-21G basis set $[76,77]$ together with the Hartree-Fock (HF) method, since this basis set is the smallest of its kind but provides reasonable geometries and energies. It is well known that HF, due to the lack of electron correlation, increases the barrier heights between conformers (an effect that becomes more acute with large basis sets, as for intance 6-31G*) but HF/3$21 \mathrm{G}$ tends to correct that effect by error compensation. Another important aspect is that HF/3-21G is superior to the traditional semiempirical methods when accounting for hydrogen bond interactions [78]. This is an important aspect because in molecules presenting $-\mathrm{OH}$ and $-\mathrm{NH}_{2}$ groups, some of the most stable conformers may contain strong hydrogen bonds. More recent semiempirical [79] and tight-binding methods [80] have improved this and other aspects by which they traditionally failed, so they are also viable options as LL methods. However, in a recent work, Mancini et al. [11] tested a few semiempirical methods (including PM7 [79]) against a reference set of data on threonine (Thr) [63] and none of the semiempirical methods was able to completely retrieve the conformers of the reference study. In this aspect, the HF/3-21G level produced 68 conformers for Thr versus the $56 \mathrm{HL}$ conformers of the reference data.

It is noteworthy that HF methods tend to generate more minima than electron correlation methods but the spurious minima disappear at the HL optimization. However, adopting HF as the LL method forced us to find efficient sampling strategies that accomplish the challenge 


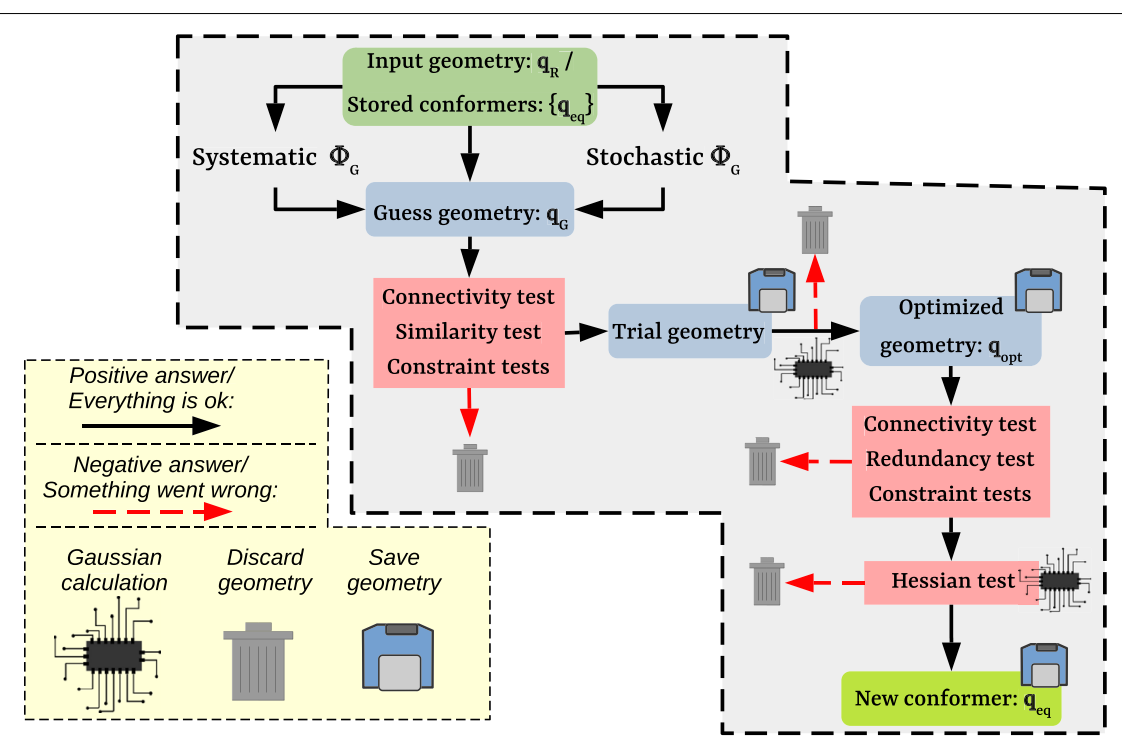

Fig. 1 Flowchart for the LL conformational search algorithm as implemented in TorsiFlex

of exploring the PES within tractable limits. In TorsiFlex, this involved the design of a battery of tests that avoid ill-generated geometries and different types of redundancies, as detailed in the next Section.

The HL calculations, that also require geometry optimizations starting from the converged LL structures and Hessian calculation over the final geometries, were performed at the M08-HX functional [81] in combination with the MG3S [82] basis set (same as MG3 [83] but with improvements for molecules containing sulfur atoms). This global-hybrid-meta-GGA functional has been specially designed for thermochemistry, kinetics, and noncovalent interactions [84-86]. For the evaluation of the partition functions, all frequencies were scaled by the recommended scale factor of 0.973 [87].

Additionally, a comparison of the current M08-HX/ MG3S calculations for the eleven most stable conformers of cysteine (Cys) against the benchmark $\operatorname{CCSD}(\mathrm{T})$ energies carried out by Wilke et al. [62] who also employed the HF/3-21G method as LL searching method, shows that the mean unsigned error (MUE) of M08-HX is only $0.25 \mathrm{kcal} / \mathrm{mol}$, whereas B3LYP//aug-cc-pVTZ yields a MUE of $0.36 \mathrm{kcal} / \mathrm{mol}$.

Our final dataset contains a total of 6508 confirmed minima obtained at the M08-HX/MG3s level. This information is available in GitHub [88] and as Additional file 1 accompanying this work. Additionally, this file contains the zero-point energy, rotational constants, and dipole moments of each conformer.

\section{Algorithm and Implementation}

Figure 1 schematically illustrates the design of the search algorithm implemented in Torsiflex. The algorithm requires an initial geometry to work with, termed the reference (or input) geometry ("Reference geometry and connectivity" Section). This geometry is the working framework to generate structures (Generation of geometries for sampling" Section) along the configurational space. However, they are validated through a series of tests ("Validation tests for the initial geometries" Section) before proceeding with the electronic structure calculations. The geometries that fail the tests are discarded, whereas those that pass them turn into trial structures that are optimized. In this manner, Torsiflex avoids unnecessary calculations, accelerating the search process. If the optimization succeeds, the resulting geometry is treated as a potential conformer, condition that is verified by submitting the structure to a new set of tests ("Validation of the optimized geometry" Section). Torsiflex also accounts for amine inversion ("Umbrella inversion of the $\mathrm{NH}_{2}$ group" Section), and conformational enantiomerism ("Conformational enantiomers" Section). Finally, the parallelization of the search algorithm ("Parallelization within the search algorithm" Section) and the duallevel method ("Dual-level approach" Section) are also discussed. 

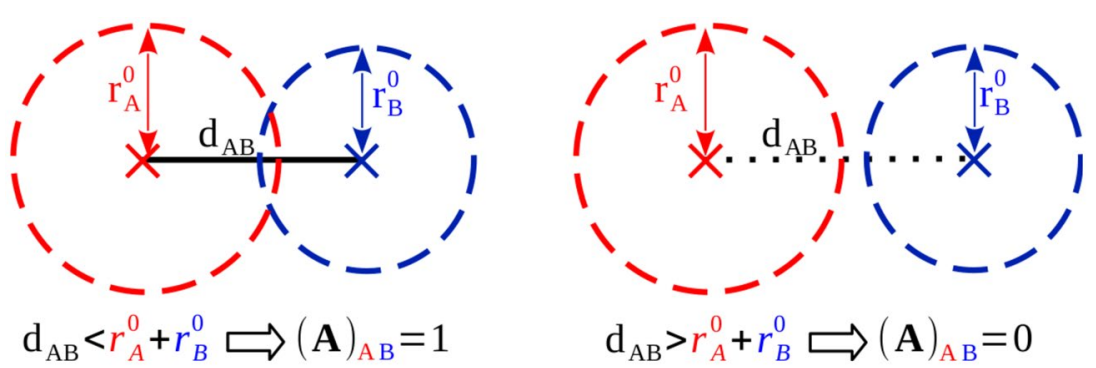

Fig. 2 Bonding criterium for a pair of atoms, $A$ and $B$

\section{Reference geometry and connectivity}

Torsiflex reads the reference geometry in internal coordinates, $\mathbf{q}_{\mathrm{R}}$, employing the Z-matrix format. ${ }^{1}$ These internal coordinates should explicitly include the $K$ proper torsions of interest univocally defined, i.e. the torsion about a given single bond can only be defined once. ${ }^{2}$ The reference geometry, in addition to its role in the generation of the first candidates in the search process, defines the molecular connectivity of the system, represented by its adjacency (or connectivity) matrix, $\mathbf{A}_{\mathrm{R}}$. This square matrix is employed to build finite graphs and its elements reveal whether pairs of nodes (atoms) are adjacent (bonded) or nonadjacent (not bonded) in the graph (molecule). For a system with $N$ atoms, $\mathbf{A}_{\mathrm{R}}$ is a $N \times N$ symmetric square matrix where each element is given by:

$$
\left(\mathbf{A}_{\mathrm{R}}\right)_{i j}=\left\{\begin{array}{cc}
1 & \text { if } \text { bonded and } i \neq j \\
0 & \text { otherwise }
\end{array}\right.
$$

Torsiflex considers a pair of atoms (A, B) to be bonded when the distance between them, $d_{A B}$, is smaller than the sum of their corresponding covalent radii, $r_{A}^{0}$ and $r_{B}^{0}$,

$$
d_{A B}<\left(r_{A}^{0}+r_{B}^{0}\right)
$$

as illustrated in Fig. 2. In particular, TorsiFlex applies the criterium of Eq. 2, but considering a scale factor, $f_{\mathrm{c}}$, that facilitates the control of this connectivity by the user. Specifically, the program assumes that a pair of atoms $(\mathrm{A}, \mathrm{B})$ is bonded when:

$$
d_{A B}<f_{\mathrm{c}} \cdot\left(r_{A}^{0}+r_{B}^{0}\right)
$$

In this inequality, a larger $f_{\mathrm{c}}$ value increases the probability for two atoms to be considered as bonded.

\footnotetext{
${ }^{1}$ All reference Z-matrix geometries for the 20 amino acids are listed in the Additional file 1 .

2 As for example, in Gly, the target torsion about the $\mathrm{C}-\mathrm{C}$ single bond can be defined as N-C-C-O. However, the two methylene $\mathrm{H}_{1}$ and $\mathrm{H}_{2}$ atoms need to be specified as improper torsions (e.g. $\mathrm{H}_{1}-\mathrm{C}-\mathrm{N}-\mathrm{C}$, and $\mathrm{H}_{2}-\mathrm{C}-\mathrm{N}-\mathrm{C}$ ).
}

The adjacency matrix avoids the optimization of highly unstable geometries and discriminates between constitutional isomers (i.e. structures with different bonding patterns) [89]. An example of this type of isomerism can be found in the canonical and zwitterionic forms of general amino acids, both represented by different adjacency matrix (see Fig. 3).

A similar situation may appear if two atoms are close enough to be spuriously considered as bonded by Eq. 3, but such undesired arrangements are also discarded by applying this connectivity test.

\section{Generation of geometries for sampling}

Torsiflex builds a $K$-dimensional torsional vector, $\boldsymbol{\Phi}^{\mathrm{R}}=\left(\phi_{1}, \ldots, \phi_{i}, \ldots, \phi_{\mathrm{K}}\right)$ from the target torsions specified in the reference geometry. Therefore, this vector is contained in the internal coordinates definition of the Z-matrix:

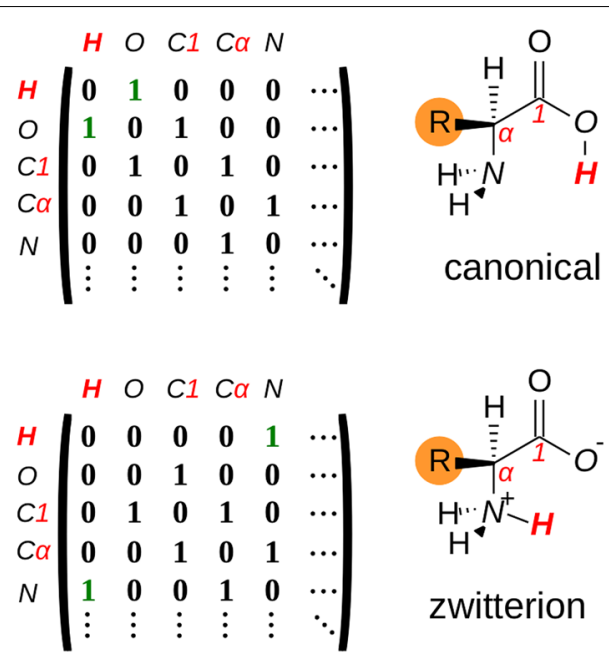

Fig. 3 Adjacency matrices of the canonical and zwitterion forms for a general amino acid. The numbering of carbon atoms follows the IUPAC \& IUB recommendation [90] 


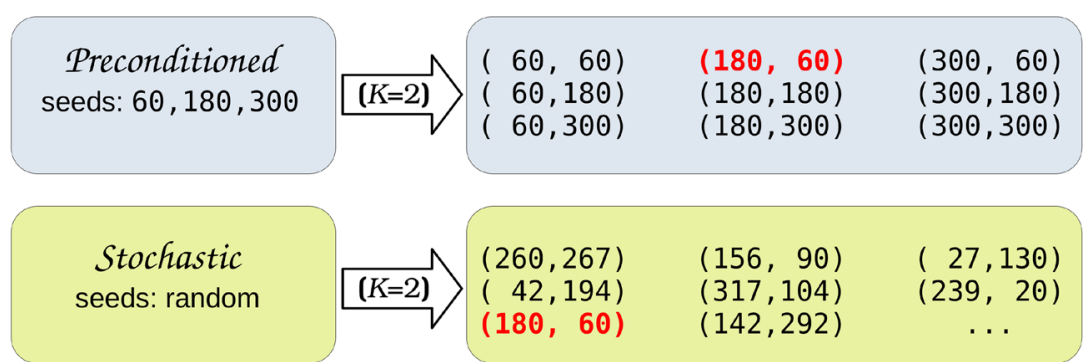

Fig. 4 Preconditioned and stochastic generation of torsional vectors for a system with two torsions $(K=2)$. Seeds in the preconditioned structures correspond to the two gauche and anti conformations. The bold-red vector that appears in the preconditioned and stochastic searches is skipped in the latter. Ellipsis in the stochastic algorithm indicates that we can generate as many guesses as desired

$$
\boldsymbol{\Phi}^{\mathrm{R}} \subset \mathbf{q}_{\mathrm{R}}
$$

and its replacement by a vector with guessed dihedral angles, $\boldsymbol{\Phi}^{\mathrm{G}}$, renders an initial geometry, $\mathbf{q}_{\mathrm{G}}$ (i.e. the dihedral angles in the reference Z-matrix are modified). In mathematical form:

$$
\mathbf{q}_{\mathrm{G}}=\left(\mathbf{q}_{\mathrm{R}} \backslash \boldsymbol{\Phi}^{\mathrm{R}}\right) \cup \boldsymbol{\Phi}^{\mathrm{G}}
$$

In practice, Torsiflex operates with the structural parameters of the previously optimized geometries instead of resorting to the reference Z-matrix. Thus, the Z-matrix of the new structure is generated from that of the closest optimized conformer but substituting the dihedral angles of the target torsions by the new guessed ones. This strategy pursues two goals: (i) the improvement of the starting geometry by employing more suitable bond lengths, bond angles, and improper torsions than those of the reference geometry, and (ii) the speed-up of geometry optimizations. Tradicionally, many algorithms based on systematic searches employ exclusively the reference geometry in the generation of new structures, an approach sometimes referred as 'rigid rotor' approximation [18]. However, this update of the geometric parameters gains relevance as the size of the torsional vector increases. Otherwise, we run the risk of leaving important parts of the potential energy surface unexplored, since the reference values of the $3 N-K-6$ degrees of freedom ( $N$ is the number of atoms) may be substantially far from the equilibrium configuration that matches a particular torsional vector.

The vectors employed as initial guesses, $\boldsymbol{\Phi}^{\mathrm{G}}$, can be generated in two different fashions: preconditionally or stochastically (see Fig. 4). The former is based on chemically intuitive dihedral angles as, for example, the expected gauche $\left(60^{\circ}\right.$ and $\left.300^{\circ}\right)$ and anti $\left(180^{\circ}\right)$ configurations in $s p^{3}$ carbon atom chains or the syn $\left(0^{\circ}\right)$ and anti $\left(180^{\circ}\right)$ arrangements of carboxylic acids, respectively.
The latter is based on the random generation of torsional angles and may lead to values already sampled, as indicated in Fig. 4. A similarity test needs to be performed before starting the optimization of the geometry to avoid this redundancy.

\section{Validation tests for the initial geometries}

The initial geometry generated from a preconditioned or a stochastic seed, $\mathbf{q}_{\mathrm{G}}$, needs to be geometrically optimized at LL by an electronic structure program. If this optimization succeeds, the Hessian matrix of the optimized geometry, $\mathbf{q}_{\text {opt }}$, must be calculated to assert that we are indeed dealing with an equilibrium structure. These two steps should be carried out only if necessary and, for this reason, the initial geometries must overcome the series of validation tests listed below:

- Connectivity test. The initial geometry should contain the same adjacency matrix as the reference

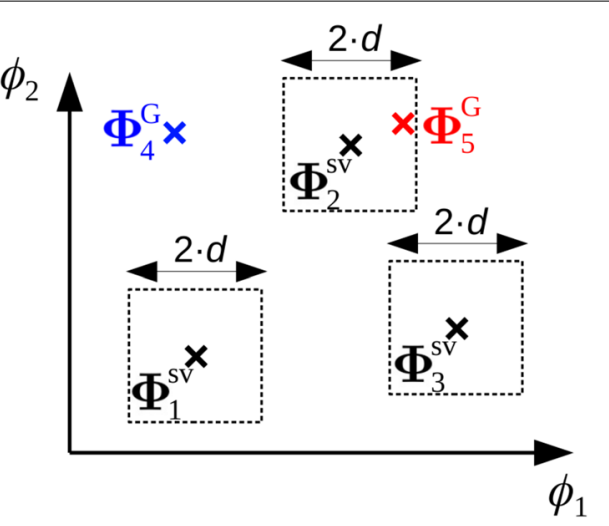

Fig. 5 Scheme for the similarity test in a system with $K=2$. The test results positive for vector $\boldsymbol{\Phi}_{4}^{G}$. On the contrary, the test is negative for the fifth guess point, $\boldsymbol{\Phi}_{5}^{G}$, because it is inside the domain of the second saved point, $\boldsymbol{\Phi}_{2}^{\text {sv }}$ 
geometry. Thus, if $\mathbf{A}_{\mathrm{G}}$ is the adjacency matrix associated with $\mathbf{q}_{\mathbf{G}}$, this test is positive if:

$$
\mathbf{A}_{\mathrm{R}}=\mathbf{A}_{\mathrm{G}}
$$

- Similarity test. The torsional vector of the generated structure, $\boldsymbol{\Phi}^{\mathrm{G}}$, is compared with a pool of saved torsional vectors from previous iterations, $\left\{\boldsymbol{\Phi}^{\mathrm{sv}}\right\}$; if $\boldsymbol{\Phi}^{\mathrm{G}}$ falls outside of the domain $d$ that surrounds each stored vector, then the test is positive (see Fig. 5). Mathematically, this condition can be written as:

$$
\forall p, \exists \tau:\left|\left(\boldsymbol{\Phi}^{\mathrm{G}}\right)_{\tau}-\left(\boldsymbol{\Phi}_{p}^{\mathrm{sv}}\right)_{\tau}\right|>d
$$

where $p$ and $\tau$ run over all saved points and over each target torsion, respectively. The domain associated with the stored values is provided by the user and by default is $d=15^{\circ}$.

- Constraints test. For different reasons, the user may be interested in a set of conformers presenting certain characteristics. For example, the conformers with the hydrogen of the carboxylic group (dihedral $\mathrm{O}=\mathrm{C} 1-\mathrm{O}-\mathrm{H}$ ) in anti position (dihedral angle in the $150^{\circ}-210^{\circ}$ interval), and with the distance between the $\mathrm{O}-\mathrm{H}$ and the $\mathrm{N}$ atom of the amino group smaller than $2.5 \AA$. For such situations, TorsiFlex accepts the definition of constraints based on the distance between a pair of atoms, the angle between a triad of atoms and the dihedral angle between four atoms. Specifically, Torsiflex differenciates between two types of constraints:

- Hard constraints: the test is positive if all of the defined constraints are fulfilled.

- Soft constraints: the tests is positive if at least one of the defined constraints is fulfilled.

If any of the previous tests delivers a negative result, Torsiflex discards $\mathbf{q}_{\mathrm{G}}$. Therefore, LL optimizations that could lead to constitutional isomers, previously located conformers, or undesired conformers, are skipped, speeding up the searching process. The initial geometry that passes all tests turns into a trial geometry, ready to be optimized.

\section{Validation of the optimized geometry}

Optimum performance of the search algorithm is achieved when the preconditioned generation of structures precedes the stochastic one for two reasons. Firstly, structure optimizations from preconditioned seeds rarely fail and, secondly, initial geometries with different preconditioned seeds infrequently lead to the same conformer. Geometries built from random seeds have the disadvantage that are alien to any chemical characteristic of the system and should be our second choice. However, they are crucial to improve the sampling of the PES, mainly to access conformers that involve chemical interactions that went unnoticed in the first screening.

Beyond the optimum performance of the algorithm, the aforementioned order also enables estimating the importance of the chemically intuitive conformers. Particularly, Torsiflex classifies the located conformers depending on whether they were obtained from preconditioned or stochastic seeds. Such classification can be used to estimate the importance of the chemically-intuitive conformers, associated with the preconditioned search.

Once a trial geometry is optimized, the resulting geometry, $\mathbf{q}_{\text {opt }}$, is available. In order to assert if $\mathbf{q}_{\text {opt }}$ corresponds to a new conformer, a second set of tests is sequentially executed:

- Connectivity test. It ensures that the optimized geometry still represents the original constitutional isomer by comparing its adjacency matrix $\mathbf{A}_{\text {opt }}$ with the reference one:

$$
\mathbf{A}_{\mathrm{R}}=\mathbf{A}_{\mathrm{opt}}
$$

- Redundancy test. It compares the optimized torsion vector, $\boldsymbol{\Phi}^{\text {opt }}$, against the pool of saved equilibrium structures, $\left\{\boldsymbol{\Phi}^{\mathrm{eq}}\right\}$. If $\boldsymbol{\Phi}^{\mathrm{opt}}$ is missing from $\left\{\boldsymbol{\Phi}^{\mathrm{eq}}\right\}$, i.e., if $\boldsymbol{\Phi}^{\text {opt }} \not \subset\left\{\boldsymbol{\Phi}^{\mathrm{eq}}\right\}$, the test is positive and the optimized geometry is a new conformer incorporated into $\left\{\boldsymbol{\Phi}^{\mathrm{eq}}\right\}$. However, to account for numerical errors, this test is considered positive when:

$$
\forall p, \exists \tau:\left|\left(\Phi^{\mathrm{opt}}\right)_{\tau}-\left(\boldsymbol{\Phi}_{p}^{\mathrm{eq}}\right)_{\tau}\right|>\epsilon
$$

with $\epsilon$ being not greater than 2 degrees. This test is essentially equivalent to the similarity test, but it is controlled by a different threshold $(\epsilon<d)$ and the comparison is against the located conformers instead of all the previously saved vectors.

- Constraints test. Torsiflex verifies the hard and soft constraints also on the optimized geometry.

- Hessian test. It inspects the vibrational frequencies resulting from the diagonalization of the Hessian matrix of the LL optimized geometry. If all vibrational frequencies are real, the test is positive. ${ }^{3}$

If any of the first three tests is negative, $\mathbf{q}_{\text {opt }}$ is automatically discarded and no computer time is wasted carrying out the Hessian test. The validation of initial geometries

\footnotetext{
${ }^{3}$ When dealing with the conformers of a transition state, the test is positive when all frequencies but one (which is imaginary) are real.
} 


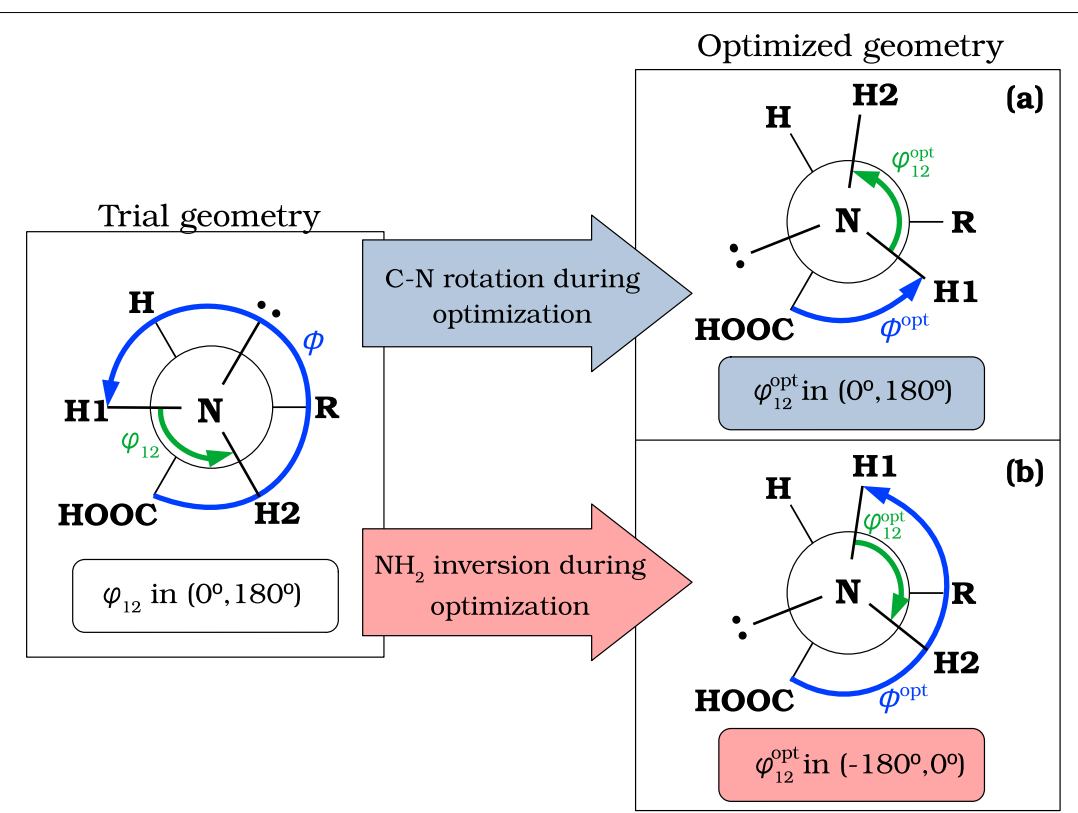

Fig. 6 Umbrella inversion motion of $\mathrm{NH}_{2}$ belonging to a generic amino acid backbone. The torsional angle $\phi=\mathrm{C} 1-\mathrm{C}_{\alpha}-\mathrm{N}-\mathrm{H} 1$ differs in structures $\mathbf{a}$ and $\mathbf{b}$ but the structure is the same. The improper torsion $\varphi_{12}$ of structure $\mathbf{b}$ changed sign during the optimization in such a way that $\varphi_{12}^{\mathrm{opt},(\mathrm{a})}=-\varphi_{12}^{\mathrm{opt},(\mathrm{b})}$. Therefore structure $\mathbf{b}$ can be transformed into structure $\mathbf{a}$ by exchanging $\mathrm{H} 1$ and $\mathrm{H} 2$

is only carried out at the LL, whereas the validation of the optimized geometries is performed at both, LL and HL.

Finally, we highlight that along the search process (Fig. 1), all torsional vectors associated with the initial and optimized geometries are saved in $\left\{\boldsymbol{\Phi}^{\mathrm{sv}}\right\}$. This storage, combined with the similarity test, prevents visiting the same region of the torsional space twice when performing the stochastic sampling.

\section{Umbrella inversion of the $\mathrm{NH}_{2}$ group}

The umbrella inversion, an internal motion commonly ascribed to the amino group, may interfere with the internal rotation, as both motions present low barriers. The effect of this inversion is illustrated in Fig. 6, showing that the optimization of the trial geometry of a general amino acid may follow two different paths. Structure (a) arises from the internal rotation about the $\mathrm{C}_{\alpha}-\mathrm{N}$ bond, whereas structure $\mathbf{b}$ is obtained as a result of umbrella inversion motion. Both structures correspond to the same conformer but the dihedral angle that describes the proper torsion about the $\mathrm{C}_{\alpha}-\mathrm{N}$ group, $\phi=\mathrm{C} 1-\mathrm{C}_{\alpha}-\mathrm{N}-\mathrm{H} 1$, differs from a to $\mathbf{b}$. Therefore, the optimized value of $\phi$, without any additional information, provides an ambiguous description of the $\mathrm{NH}_{2}$ internal motion.

For this reason, Torsiflex automatically monitors the $\varphi_{12}=\mathrm{H} 1-\mathrm{H} 2-\mathrm{C}_{\alpha}-\mathrm{N}$ improper torsion that changes sign upon umbrella inversion. ${ }^{4}$ Specifically, Torsiflex

\footnotetext{
${ }^{4}$ Assuming that dihedral angles are defined between $-180^{\circ}$ and $+180^{\circ}$
}

compares the improper torsion at both, the trial $\varphi_{12}$ and the optimized $\varphi_{12}^{\text {opt }}$ structures: if $\operatorname{sgn}\left(\varphi_{12}\right)=\operatorname{sgn}\left(\varphi_{12}^{\mathrm{opt}}\right)$ then no inversion occurred [structure a of Fig. 6], if $\operatorname{sgn}\left(\varphi_{12}\right) \neq \operatorname{sgn}\left(\varphi_{12}^{\text {opt }}\right)$ then the inversion took place during one optimization step [structure b of Fig. 6], and Torsiflex exchanges the $\mathrm{H} 1$ and $\mathrm{H} 2$ hydrogen atoms. In summary, $\phi$ can be used as the torsional target in the amino group but only after verifying that $\varphi_{12}$ preserves its sign in the optimized geometries.

\section{Conformational enantiomers}

Some flexible molecules have a plane of symmetry for specific values of their dihedral angles; such molecules exhibit conformational enantiomerism. In the case of pAAs, only glycine (Gly) possesses this type of enantiomers, due to the absence of asymmetric carbon atoms. As a consequence of the plane of symmetry, only half of the torsional space needs to be explored.

For a given geometry defined by the torsional vector, $\boldsymbol{\Phi}=\left(\phi_{1}, \ldots, \phi_{i}, \ldots, \phi_{\mathrm{K}}\right)$, Torsiflex retrieves the vector associated with the conformational enantiomer, $\boldsymbol{\Phi}^{*}=\left(\phi_{1}^{*}, \ldots, \phi_{i}^{*}, \ldots, \phi_{\mathrm{K}}^{*}\right)$, and stores it together with $\boldsymbol{\Phi}$. The similarity and redundancy tests take advantage of this double storage, reducing the computer time. The torsional angles of the enantiomer can be calculated considering that for the configuration of $C_{s}$ symmetry, there are target torsions: (i) with the 4 atoms lying in the plane of symmetry or (ii) with one or more atoms out of the plane. 


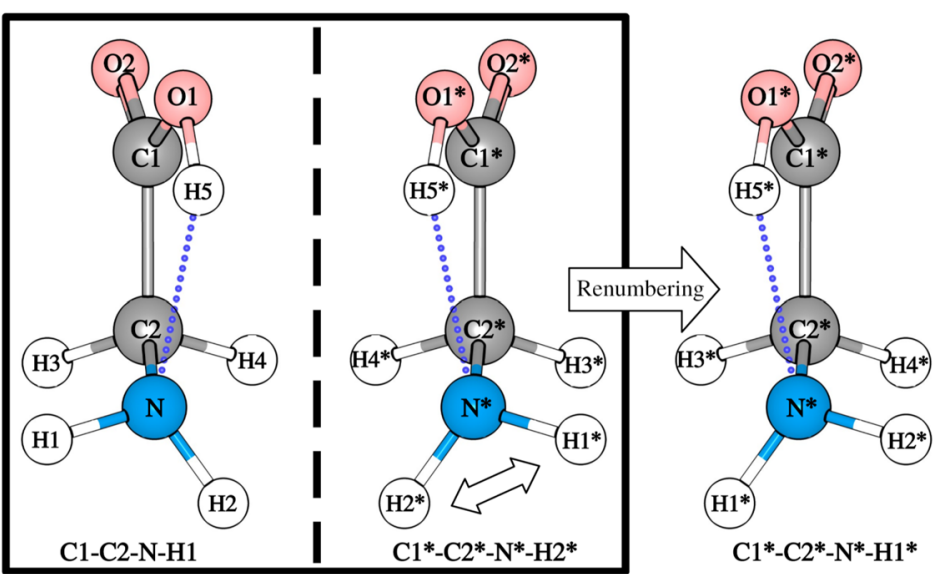

Fig. 7 Enantiomer generation and renumbering in glycine. Notice that $\mathrm{C} 2$ is the $\mathrm{C}_{\alpha}$ carbon.

In case (i), for a given structure with torsion $\phi_{i}$, the torsional angle of the enantioner is simply

$$
\phi_{i}^{*}=-\phi_{i}
$$

In case (ii), the out-of-plane atoms have replicas at the other side of the plane, and in addition to the dihedral angle of the four atoms, $\phi_{i}$, we need to know the dihedral angle $\bar{\phi}_{i}$ between the atoms that are identical under reflection. The relation is:

$$
\phi_{i}^{*}=-\bar{\phi}_{i} ; \quad \bar{\phi}_{i}^{*}=-\phi_{i}
$$

For the case of Gly displayed in Figure 7, the target torsional angles are $\boldsymbol{\Phi}=\left(\phi_{1}, \phi_{2}, \phi_{3}\right)$, where $\phi_{1}=\mathrm{H} 5-\mathrm{O} 1-\mathrm{C} 1$ $\mathrm{C} 2, \quad \phi_{2}=\mathrm{O} 1-\mathrm{C} 1-\mathrm{C} 2-\mathrm{N}, \quad \phi_{3}=\mathrm{C} 1-\mathrm{C} 2-\mathrm{N}-\mathrm{H} 1, \quad$ and $\bar{\phi}_{3}$ $=\mathrm{C} 1-\mathrm{C} 2-\mathrm{N}-\mathrm{H} 2$. With the rules of Eqs. 10 and 11, the torsional set of dihedrals of the enantiomer corresponds to $\boldsymbol{\Phi}^{*}=\left(-\phi_{1},-\phi_{2},-\bar{\phi}_{3}\right)$.

In practice, for a given optimized structure TorsiFlex generates the enantiomer by projecting the geometry onto the $Y Z$ plane, i.e, by changing the sign of the $x$ Cartesian coordinate of every atom. The reflection on the plane generates a non-superimposable structure (that cannot be obtained by internal rotations) with respect to the original structure, as shown in Fig. 7. However, conformational enantiomers can interconvert between them, and some atoms of the reflected structure need to be renumbered to meet this condition.

Torsiflex renumbers the atoms in three steps:

1 Correlation of atoms of the same type. For example, $\mathrm{H} 1^{*}$ can only be correlated to $\mathrm{H}$ atoms in the original structure, i.e. H1 to H5. In this step, the $\mathrm{N}$ atom is automatically correlated.
2 Inspection of the connectivity. As $\mathrm{H} 1^{*}$ is bonded to the nitrogen atom, it can only correlate to $\mathrm{H} 1$ and H2. In this step, Torsiflex also correlates (univocally) $\mathrm{C}^{*}, \mathrm{C} 2^{*}, \mathrm{O}^{*}, \mathrm{O} 2^{*}$ and $\mathrm{H} 5^{*}$.

3 Inspection of the spatial distribution through improper torsions. The dihedral angle associated with the improper torsion $\varphi_{12}^{*}=\mathrm{H} 1^{*}-\mathrm{H} 2^{*}-\mathrm{N}^{*}-\mathrm{C} 2^{*}$ is compared against the $\varphi_{12}=\mathrm{H} 1-\mathrm{H} 2-\mathrm{N}-\mathrm{C} 2$, and $\varphi_{21}$ $=\mathrm{H} 2-\mathrm{H} 1-\mathrm{N}-\mathrm{C} 2$ dihedral angles of the original structure. The improper torsional angles have the following property,

$$
\varphi_{12}=-\varphi_{21}
$$

but are invariant under reflection. Therefore, the correlation between improper torsions is $\varphi_{12}=\varphi_{21}^{*}$ and $\varphi_{21}=\varphi_{12}^{*}$. This relation allows an unambiguous assignment of $\mathrm{H}_{2}^{*}$ to $\mathrm{H} 1$ and $\mathrm{H} 1^{*}$ to $\mathrm{H} 2$. Finally, $\mathrm{H} 2^{*}$ and $\mathrm{H}_{1}^{*}$ are renumbered as $\mathrm{H}^{*}$ and $\mathrm{H} 2^{*}$, respectively, to preserve the same numbering as in the original structure. The same procedure can be applied to $\mathrm{H} 3$ and $\mathrm{H} 4$.

After the correct assignment of all atoms of the enantiomer, $\boldsymbol{\Phi}^{*}$ is readily calculated. Notice that the above procedure reduces by half the computational effort for molecules presenting conformational enantiomerism.

\section{Parallelization within the search algorithm}

The previous algorithm for systems with several torsions may be computationally expensive when resorting to LL ab initio methods (or even semi-empirical methods, depending on the number of torsions). Torsiflex allows different batches to be executed at the same time, achieving in this manner an artificial 100\% parallelization. 
The standard execution for a preconditioned search is:

python3 torsiflex.py --prec

which sequentially generates the initial geometries. However, this procedure may be too slow in systems with $K>3$. In order to speed up the search, Torsiflex can split the set of seeds into $M$ groups $\left\{n_{1}, n_{2}, \ldots, n_{\mathrm{m}}, \ldots, n_{\mathrm{M}}\right\}$ and deal with each of them separately. Torsiflex can manage the $m$-th group $(m \leq M)$ by:

python3 torsiflex.py --prec M m

For example, to divide the seeds into $M=10$ groups and to perform calculations on the elements $n_{2}(\mathrm{~m}=2)$ the command line is:

python3 torsiflex.py --prec 102

Regarding the stochastic search, each execution generates random seeds, and therefore, different batches can be carried out simultaneously. The number of seeds generated in each execution within the stochastic search flow can be controlled in the input file.

\section{Dual-level approach}

The dual-level approach consists on performing more accurate HL electronic structure calculations employing as trial the set of structures of the already known LL equilibrium geometries. As for the LL case, each HL optimized geometry must overcome the validation tests ("Validation of the optimized geometry" Section) to be stored as a new conformer of the system. The dual-level method is a double-edged sword, a claim that can be extended to all methods that rely on PES that are sampled at LL. On the one hand, it plays a key role in accelerating the search process but, on the other hand, it may miss conformers existing in the HL PES. For this reason, it is of capital importance to select a LL torsional PES characterized by a similar topology than that of the HL torsional PES.

We have found that HF/3-21G calculations are a good compromise for this case. This method is fast enough and tends to produce more minima than electronic correlated methods. Unfortunately, the size of the molecular system or the availability of computational resources can render even HF unfeasible. In such cases, MM or semiempirical methods may be the only choice. However, even in the most favorable situation, it is almost impossible to assure that a given LL PES will completely map the HL PES without loss of conformers.

\section{Results and discussion}

This section summarizes the results for the twenty proteinogenic amino acids. Firstly, we compare our results with previous works. Secondly, we briefly analyze the
Table 1 Number of canonical conformers obtained for the 20 pAAs employing Torsiflex

\begin{tabular}{llll}
\hline Amino acid & HF & DFT & Number of conformers [Ref.] \\
\hline Asp & 169 & 127 & $139[58], 37[70], 76[71]$ \\
Glu & 513 & 415 & $143[70], 165[69], 197[71]$ \\
Ala & 11 & 11 & $10[49], 13[50], 9[70], 15[71]$ \\
Gly & 12 & 9 & $8[60,71]$ \\
Ile & 114 & 95 & $12[65], 59[70], 76[71]$ \\
Leu & 130 & 95 & $8[65], 53[70], 85[71]$ \\
Pro & 20 & 16 & $18[52], 3[70], 22[71]$ \\
Val & 35 & 30 & $6[65], 19[70], 22[71]$ \\
Asn & 91 & 64 & $62[55], 23[70], 49[71]$ \\
Gln & 271 & 181 & $72[70], 143[68], 134[71]$ \\
Phe & 41 & 35 & $37[57], 20[70], 25[71]$ \\
Trp & 95 & 76 & $45[53], 37[70], 57[71]$ \\
Tyr & 80 & 65 & $76[54], 38[70], 43[71]$ \\
Arg & 3199 & 2811 & $520[70], 1218[71]$ \\
HisD & 76 & 60 & $10[64], 57[71]$ \\
HisE & 93 & 70 & $15[64], 47[71]$ \\
Lys & 2224 & 1944 & $927[61], 391[70], 733[71]$ \\
Ser & 73 & 64 & $51[49], 74[66], 30[70], 59[71]$ \\
Thr & 68 & 58 & $56[63], 65[66], 29[70], 47[71]$ \\
Cys & 79 & 66 & $42[49], 71[62], 87[66], 50[70], 99[71]$ \\
Met & 271 & 216 & $27[48], 113[70], 246[71]$ \\
\hline Second & &
\end{tabular}

Second and third columns list the number of conformers obtained at the LL and $\mathrm{HL}$, respectively. The last row indicates the number of conformers obtained in previous works with the reference between square brackets. Two histidine tautomers, HisD, and HisE have been considered (also referred in literature as $\mathrm{His}\left[\mathrm{N}^{\tau} \mathrm{H}\right]$ and His $\left[\mathrm{N}^{\pi} \mathrm{H}\right]$, respectively). HF and DFT refer to the $\mathrm{HF} / 3-21 \mathrm{G}$ and M08-HX/MG3S levels, respectively

conformational hierarchy and the preferred conformations of the twenty pAAs. Finally, we discuss the importance of the stochastic search.

\section{Comparison with previous works}

Table 1 contains the number of conformers located for each pAA using TorsiFlex, including both the number of conformers located in the LL search (7 665 in total) and the resulting conformers after the HL refinement (6 508). We notice that around $85 \%$ of the LL conformers led to a new conformer in the M08-HX/MG3S PES. These results do not correlate completely due to the HF tendency of producing more minima than electronic correlated methods.

The most complete work on conformations of pAAs has been carried out by Ropo et al. [71], but their numbers do not correspond exclusively to conformers (minima), but rather to stationary points in the PES, as they pointed out in their work: "The present data contains stationary-point geometries (mainly minima, but also saddle points since no routine normal-mode analysis was performed) on the potential energy surface of the 20 proteinogenic amino 


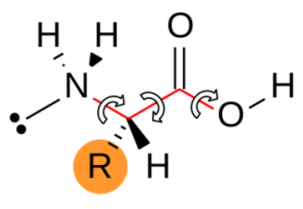

Type I

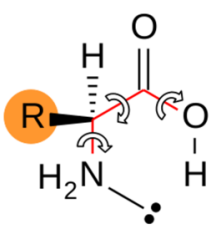

Type II

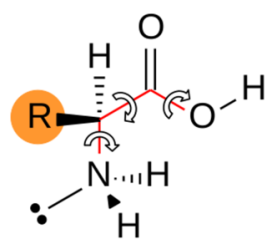

Type III

Fig. 8 Classification of amino acids backbones. The torsional bonds that describe types I, II, and III are highlighted in red

acids" [71]. In this sense, their numbers are an upper limit to the total number of conformers. For example, they have found a total of 246 stationary points for methionine (Met), whereas we have located 216 conformers; this difference cannot be associated with a poor performance of Torsiflex for this system, as the percentage of minima in their geometries is unknown. Nevertheless, we should remark that the total number of HL conformers found with Torsiflex (6 508) surpasses their number of stationary points (3 315) by roughly a factor of two.

Regarding other works, the number of conformers located with Torsiflex in the LL search is, in general, greater than numbers previously reported for the twenty amino acids. This result still holds when we consider the number of conformers after the HL refinement with M08-HX/MG3S, with the exception of seven amino acids: Asp (127 vs. 139 [58]), Ala (11 vs. 13 [50], Pro (16 vs. 18 [52]), Phe (35 vs. 37 [57]), Ser (73 vs 74 [66]), Cys (66 vs. 87 [66]), and Tyr (65 vs. 76 [54]), although the difference in five of them is very small and may be attributed to the difference in the electronic structure calculations. It is surprising the case of Cys with 87 conformers, since Wilke et al. [62] found 71 conformers, a number which is very close to the 66 conformers located in this study. For the remaining amino acids the variation in the number of reported conformers favors Torsiflex, and in the cases of Glu, Gln, Arg, HisE, and Lys the difference is substantial.

\section{Classification of the conformers}

We have selected the three torsions highlighted in Fig. 8 to classify the amino acids based on the backbone configurations, following the same nomenclature as in Ref. [39], that is, $\mathrm{H}-\mathrm{O}-\mathrm{C}_{1}-\mathrm{C}_{\alpha}\left(\phi_{1}\right), \mathrm{O}-\mathrm{C}_{1}-\mathrm{C}_{\alpha}-\mathrm{N}\left(\phi_{2}\right)$ and $\mathrm{C}_{1}-\mathrm{C}_{\alpha}$ $-\mathrm{N}-l p\left(\phi_{3}\right), l p$ denoting the position of the $\mathrm{N}$ lone pair. According to these arrangements, we define the following types based on a subvector of $\boldsymbol{\Phi}^{\mathrm{eq}}$ given by the dihedrals $\left(\phi_{1}, \phi_{2}, \phi_{3}\right)$ :

- Type I. It is characterized by the reference $\left(\phi_{1}^{\mathrm{R}}, \phi_{2}^{\mathrm{R}}, \phi_{3}^{\mathrm{R}}\right)=(x, 180,180)$, where $x$ can take any value in the $[0,360)$ interval; in this configuration, the hydrogen(s) of the amino group is(are) directed towards the carbonylic oxygen.

- Type II. It is specified by the reference $\left(\phi_{1}^{\mathrm{R}}, \phi_{2}^{\mathrm{R}}, \phi_{3}^{\mathrm{R}}\right)=(0,0,0)$; in this arrangement, the hydrogen of the carboxylic acid is directed towards the nitrogen lone pair.

- Type III. It is associated with the reference $\left(\phi_{1}^{\mathrm{R}}, \phi_{2}^{\mathrm{R}}, \phi_{3}^{\mathrm{R}}\right)=(180,0,180)$; the hydrogen(s) of the amino group is(are) directed towards the hydroxy group, and the hydrogen of the carboxylic acid is in syn configuration.

- Type IV. It corresponds to any configuration far from the three types.

The criterion to classify the conformers is based on the Euclidean distance calculated from the reference $\left(\phi_{1}^{\mathrm{R}}, \phi_{2}^{\mathrm{R}}, \phi_{3}^{\mathrm{R}}\right)$ dihedral angles as:

$$
d_{\mathrm{J}}=\sqrt{\sum_{i=1}^{3} \min \left(\bmod \left(\phi_{i}-\phi_{i, \mathrm{~J}}^{\mathrm{R}}, 360\right), \bmod \left(\phi_{i, \mathrm{~J}}^{\mathrm{R}}-\phi_{i}, 360\right)\right)^{2}}
$$

where $\phi_{i, \mathrm{~J}}^{\mathrm{R}}$ denotes the reference dihedral angle for the $i$ th torsion in the J-th type. ${ }^{5}$ Notice that the mod operation and the min function are compulsory due to the fact that angles $\phi$ and $\phi \pm 2 \pi n$, with $n \in \mathbb{N}$, are equivalent. Thus, the conformer is assigned to the type with the smallest distance. However, if the three $d_{\mathrm{J}}$ values are greater than a given threshold, the conformer is classified as type IV. In this work we have set this threshold to 75 degrees.

The distribution of the previous three angles for all the conformers is shown in Fig. 9, together with the corresponding type classification. As expected, conformers in amino acids are not uniformly distributed among the torsional space, although this behavior is less noticeable for the $\phi_{2}$ torsion. We have encountered a total of 2794

\footnotetext{
${ }^{5}$ When $\mathrm{J}=\mathrm{I}$, the summation excludes $\phi_{1}$, because this angle can take any value.
} 


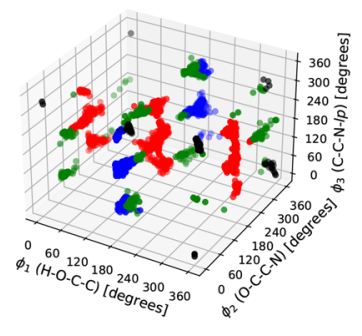

(a) 3D representation

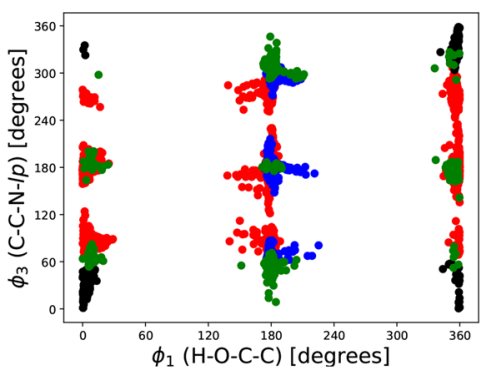

(c) 2D representation: $\phi_{1}$ vs $\phi_{3}$

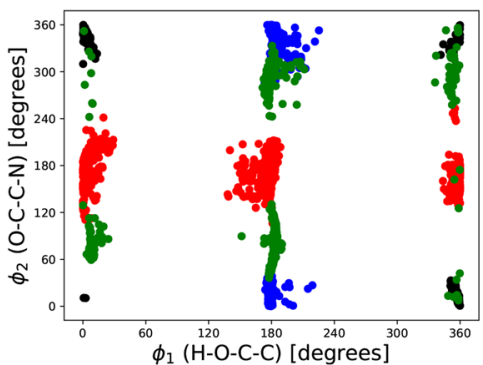

(b) 2D representation: $\phi_{1}$ vs $\phi_{2}$

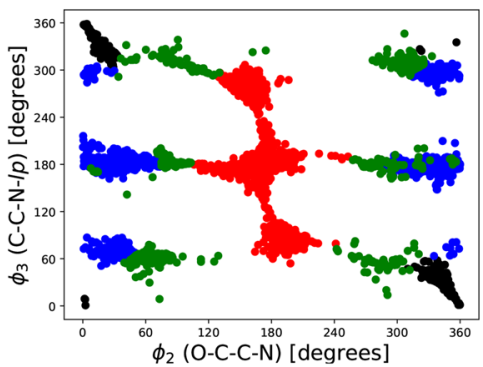

(d) 2D representation: $\phi_{2}$ vs $\phi_{3}$

Fig. 9 Distribution of conformers according to the backbone type of Fig. 8. Dihedral angles $\phi_{1}, \phi_{2}$ and $\phi_{3}$ correspond to the $\mathrm{H}-\mathrm{O}-\mathrm{C}_{1}-\mathrm{C}_{\alpha}, \mathrm{O}-\mathrm{C}_{1}-\mathrm{C}_{\alpha}-\mathrm{N}$ and $\mathrm{C}_{1}-\mathrm{C}_{\alpha}-\mathrm{N}-\mathrm{Ip}$ torsions, respectively (Ip denotes the position of the $\mathrm{N}$ lone pair). Black, red, blue and green colors identify the I, II, III and IV types, respectively

conformers of type I, 977 of type II, 1680 of type III, and 1057 of type IV.

The energy distribution of these arrangements is represented in Fig. 10. Interestingly, type I and type IV configurations are, on average, the most unstable ones, with an average relative energy of 8.03 and $8.83 \mathrm{kcal} / \mathrm{mol}$, respectively. On the contrary, type II and type III conformers are characterized by smaller relative energies (5.73 and $7.05 \mathrm{kcal} / \mathrm{mol}$ on average, respectively).

For the smallest amino acids, Gly and Ala (and also for Leu and Val), type I is the most stable arrangement, characterized by the $-\mathrm{NH}_{2} \cdots \mathrm{O}=\mathrm{C}_{1}$ hydrogen bond and by the carboxylic acid group in syn disposition. The appearance of new interactions between the $R$ fragment and the backbone for pAAs with long tails favors type II rearrangements, with a strong hydrogen bond between the nitrogen of the amino group and the hydrogen atom of the hydroxy group. The exceptions are Lys and Arg that include additional functional groups containing nitrogen at the end of the $\mathrm{R}$ fragments; these terminal nitrogen atoms form a strong hydrogen bond with the $\mathrm{OH}$ group (with the carboxylic acid group in anti disposition), leaving the amino group of the backbone in a type I arrangement. It may be argued that Asn and Gln also have terminal groups containing nitrogen in the side chain, but the interaction of the $\mathrm{OH}$ group with the acetamide seems weaker than with the amine or imine groups. For this reason, the most stable conformations of Asn and Gln are of type II.

Types I to III just account for hydrogen bonding within the common backbone. This interaction may be also of relevance between the backbone and the $\mathrm{R}$ fragment moiety, leading to geometrical distortions that are excluded from the three types. Therefore, the high number of type IV conformers is somehow expected.

In order to properly account for the significance of the conformers located during the HL search, we consider the multi-structural harmonic-oscillator (MS-HO) partition function. It takes into account all conformers and is given by [27-29]:

$$
Q_{\mathrm{rv}}^{\mathrm{MS}-\mathrm{HO}}=\sum_{j} Q_{\mathrm{rv}, j}^{\mathrm{RR}-\mathrm{HO}} e^{-U_{j} \beta}
$$

where $Q_{\mathrm{rv}, j}^{\mathrm{RR}-\mathrm{HO}}$ is the rigid-rotor harmonic-oscillator (RR-HO) rovibrational partition function of the $j$-th conformer, $U_{j}$ is its relative energy with regard to the most stable conformer and $\beta$ is $\left(k_{B} T\right)^{-1}$, with $k_{B}$ being the Boltzmann constant and $\mathrm{T}$ the temperature. Within this 

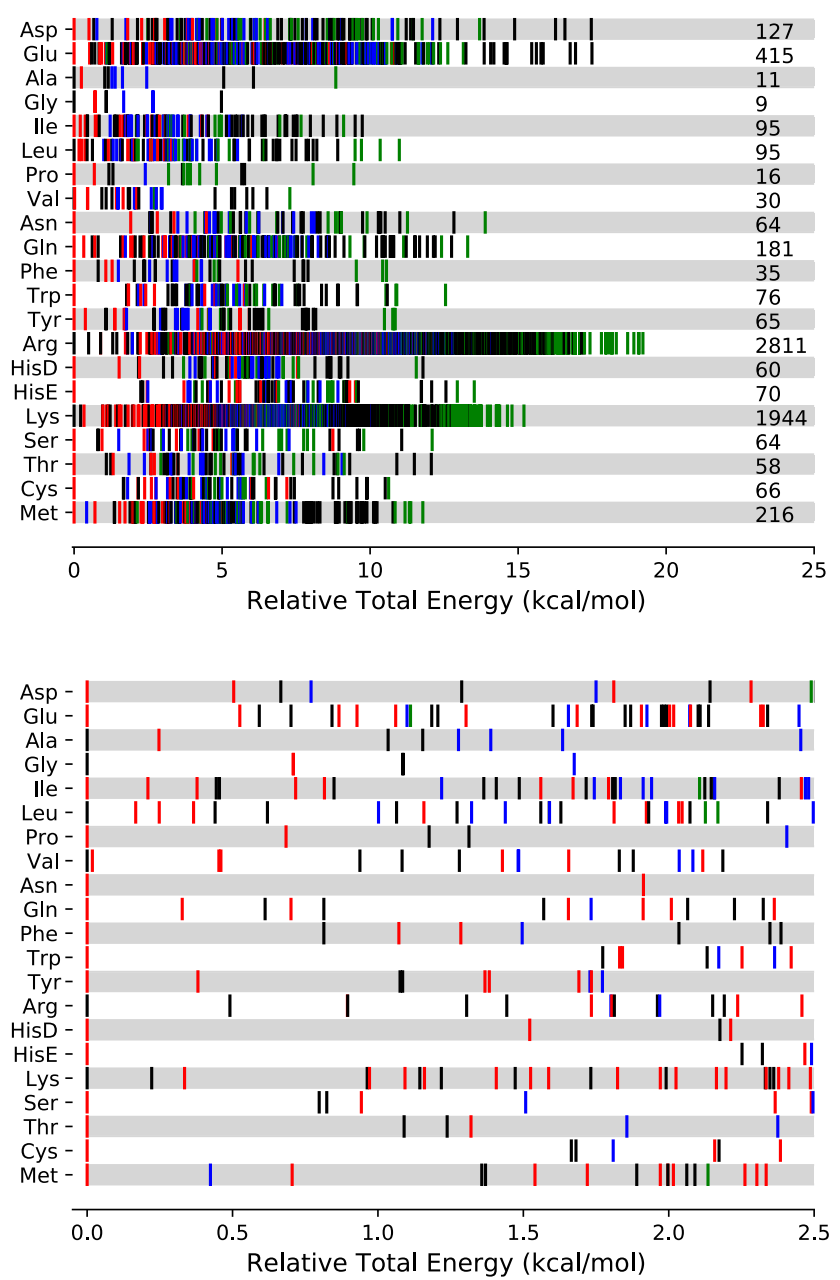

Fig. 10 Conformational hierarchies for each pAA. Type I, II, III and IV backbone configurations are represented by black, red, blue and green colors, respectively. The top figure shows the relative energy of all conformers and their total number is summarized at the end of the row. The bottom figure zooms in the most stable types within a window of $2.5 \mathrm{kcal} / \mathrm{mol}$

approximation, the contribution of a given conformer to the partition function can be obtained as:

$$
\chi_{i}(T)=\frac{Q_{\mathrm{rv}, i}^{\mathrm{RR}-\mathrm{HO}} e^{-U_{i} \beta}}{Q_{\mathrm{rv}}^{\mathrm{MS}}-\mathrm{HO}}=\frac{Q_{\mathrm{rv}, i}^{\mathrm{RR}-\mathrm{HO}} e^{-U_{i} \beta}}{\sum_{j} Q_{\mathrm{rv}, j}^{\mathrm{RR}-\mathrm{HO}} e^{-U_{j} \beta}}
$$

where we notice that this contribution is temperaturedependent and that:

$$
\sum_{i} \chi_{i}(T)=1
$$

Table 2 lists the number of conformers, sorted by their electronic energy, needed to reach 0.9 in Eq. 16, i.e., $90 \%$ of the MS-HO partition function at $300 \mathrm{~K}$. For the pAAs with few torsions the number of conformers is small (usually below 20 minima) and the largest contribution of a single conformer is well above $10 \%$, often reaching $50 \%$, and even $70 \%$ for HisD and HisE. This result justifies the common practice of considering that substantial contributions to the free energy are due to the most stable conformers in a window of 2.5 to $3.0 \mathrm{kcal} / \mathrm{mol}$ with respect to the absolute minimum, as the one showed in Fig. 10. However, in conformers with many torsional degrees of freedom, like Glu, Gln, Arg, Met, and Lys, the MS-HO partition function collects the contribution of many conformers, even if the most stable conformer contributes substantially to the partition function, as it is the case of Arg. The most notorious case is Lys for which the conformer with the largest contribution to MS-HO accounts for less than $2 \%$ of the total value. In fact, more than 600 conformations are needed to recover $90 \%$ of the partition function. This result shows that the theoretical evaluation 
Table 2 Number of conformers required to achieve $90 \%$ of the MS-HO partition function, $\mathrm{N}_{90}$, at $300 \mathrm{~K}$. The table also collects the highest relative electronic, $E^{\max }$, and free, $G^{\max }$, energies within the considered conformers. Finally, it also includes the maximum contribution of a single conformer $\left(\chi_{i}^{\max }\right)$ to the partition function

\begin{tabular}{|c|c|c|c|c|}
\hline Amino acid & $N_{90}$ & $E^{\max }$ & $G^{\max }$ & $\chi_{i}^{\max }(\%)$ \\
\hline Asp & 16 & 2.782 & 3.220 & 17.4 \\
\hline Glu & 66 & 3.312 & 4.601 & 16.9 \\
\hline Ala & 6 & 1.388 & 1.361 & 57.8 \\
\hline Gly & 5 & 1.086 & 1.138 & 57.3 \\
\hline Ile & 23 & 2.106 & 3.131 & 33.1 \\
\hline Leu & 19 & 1.931 & 3.298 & 52.4 \\
\hline Pro & 4 & 1.313 & 0.676 & 45.1 \\
\hline Val & 12 & 1.830 & 1.794 & 32.1 \\
\hline Asn & 9 & 3.266 & 1.963 & 53.1 \\
\hline Gln & 53 & 4.208 & 3.965 & 13.0 \\
\hline Phe & 10 & 2.745 & 1.542 & 28.2 \\
\hline Trp & 14 & 3.429 & 2.571 & 48.6 \\
\hline Tyr & 16 & 2.980 & 1.809 & 18.5 \\
\hline Arg & 80 & 4.333 & 5.653 & 47.3 \\
\hline HisD & 4 & 2.213 & 1.875 & 78.9 \\
\hline HisE & 5 & 2.491 & 2.261 & 70.6 \\
\hline Lys & 667 & 5.490 & 5.232 & 1.7 \\
\hline Ser & 12 & 2.627 & 2.118 & 29.6 \\
\hline Thr & 11 & 3.036 & 2.303 & 38.7 \\
\hline Cys & 16 & 3.597 & 2.708 & 31.1 \\
\hline Met & 79 & 4.120 & 3.829 & 15.0 \\
\hline
\end{tabular}

of partition functions for flexible molecules involves a thorough exploration of the torsional PES, and its characterization by just a few stable conformers may handicap the accuracy of the results.

\section{The importance of the stochastic search}

In this section we analyze and quantify the importance of the conformers located employing an stochastic algorithm, strategy avoided in many conformational studies because the search is completely random and detached from any chemical knowledge about the system. As a result, the stochastic exploration is less efficient than the preconditioned search. However, it is an ideal technique when employed after the preconditioned algorithm has already inspected the regions of the PES where conformers were expected. It allows reaching chemical structures that go beyond chemical intuition or that are stable due to unexpected intramolecular forces (like for instance hydrogen bonds between two distant fragments). When carried out in a second stage, the search is not completely random because exploits
Table 3 Contribution of the conformers encountered during the stochastic search $\chi_{\text {st }}$ (in percentage) to the MS-HO partition function at $T=300 \mathrm{~K}$

\begin{tabular}{ll}
\hline Amino acid & $\chi_{\text {st }}(\%)$ \\
\hline Asp & 0.3 \\
Glu & 1.3 \\
Ala & 0.0 \\
Gly & 0.0 \\
Ile & 3.0 \\
Leu & 0.6 \\
Pro & 0.2 \\
Val & 3.0 \\
Asn & 0.1 \\
Gln & 1.7 \\
Phe & 0.2 \\
Trp & 9.4 \\
Tyr & 0.2 \\
Arg & 3.5 \\
HisD & 0.0 \\
HisE & 0.4 \\
Lys & 1.7 \\
Ser & 0.2 \\
Thr & 0.0 \\
Cys & 0.5 \\
Met & 7.0 \\
\hline
\end{tabular}

the information supplied by the similarity test, avoiding the search across previously inspected areas.

We notice that about $10 \%$ (680) of the HL conformers appeared after the stochastic search. Although the previous percentage seems significant, it does not really quantify the importance of these conformers. Usually, they are high energy conformers that barely contribute to the macroscopic properties of the system at room temperature (see Table 3).

If the summation of Eq. 16 is restricted to the conformers associated with the stochastic search, $\chi_{\text {st }}$, we find that at $T=300 \mathrm{~K}$ this contribution is generally small, with the exception of two amino acids, Trp, and Met, for which the contribution is larger than $5 \%$, but none of the cases reached $10 \%$. These results clearly indicate that the preconditioned search already retrieves the most stable conformers and that the stochastic algorithm, at least in the case of amino acids, has a minor contribution to the partition function.

Additionally for the case of Lys, we have performed a preconditioned search at two different LL methods. At the HF/3-21G level 2224 minima were located; however, the same initial torsional angles only produced 1412 minima when the search was carried out at the PM6 [91] 
semiempirical method. These numbers point toward the same direction as the study of Mancini et al. [11] when studying Thr, as the semiempirical methods employed produced fewer conformers than the benchmark study. These results indicate that a proper selection of the LL method is critical. To this conclusion we may add that the additional conformers obtained during the stochastic search have a modest influence on the room-temperature MS-HO partition function.

\section{Limitations and future work}

In its current version, Torsiflex cannot automatically deal with different conformations arising from ring deformations, although it is possible to include them by finely tuning the torsions inside the ring (as was the case in proline). The input of the program also assumes some user skills in the construction of the Z-Matrix, which is a drawback.

Torsiflex is an open-source code written in Python 3 that can be easily modified to incorporate other electronic structure software besides Gaussian. It can be combined to structure generation programs, as for instance RDKit [92] or MAYGEN [93]. In particular, we are developing a structure-builder software that tries to avoid the definition of an initial Z-Matrix by the user. In addition to the generation of the chemical graph, this task involves, first, spotting the internal rotations, and second, modeling the rest of the structural parameters taking into account the targeted torsions. Another extension that is in our sights, is the automatic search and location of conformations as a result of ring puckering in 5, 6 and 7 member rings.

\section{Conclusions}

We have presented a new program, Torsiflex, intended for the search of conformers of acyclic molecules with several torsional degrees of freedom. The proposed algorithm is based on the synergy between preconditioned and stochastic methods at an inexpensive level that, combined with a dual-level approach, is able to find the conformers of the torsional PES at a high level method. Torsiflex automatizes the whole process and almost no actions are required from the user. The combination of a battery of validation tests together with a suitable low-level electronic structure method accelerates the searching process, making it more efficient. Additionally, issues like umbrella inversion and conformational enantiomerism are automatically taken into account by the program.

In order to show the effectiveness and capabilities of this software, we have employed Torsiflex to automatically locate the conformers of the twenty proteinogenic amino acids. Within the dual-level approximation we have employed the HF/3-21G as a low-level method and the M08HX/MG3S method as the high level. A total of 6508 conformers were found, a number that improves substantially the total amount of previously reported conformers. For the most flexible amino acids a large number of conformers is required to recover $90 \%$ of the MS-HO partition function. The results also indicate that, at least in the case of the amino acids, a well designed preconditioned search suffices to obtain converged MS-HO partition functions and that search of new conformers through a stochastic search is less important than the choice of the low-level method adopted for the exploration of the PES.

\section{Availability and requirements}

Project name: Torsiflex (version 2021.3)

Project home page: https://github.com/cathedralp $\mathrm{kg} /$ TorsiFlex

Operating systems: Linux and MacOS

Programing language: Python 3

Other requirements: None

License: MIT

\section{Supplementary Information}

The online version contains supplementary material available at https://doi. org/10.1186/s13321-021-00578-0.

Additional file 1. It contains for each of the pAAs: the Z-matrix for the reference geometry indicating the target torsions, the M08-HX/MG3S absolute energy of the most stable conformer, the relative energy, moments of inertia, dipole moments, zero-point energy, normal mode vibrational frequencies and Cartesian coordinates of the M08-HX/MG3S optimized geometries of each conformer.

\section{Acknowledgements}

The authors thank "Centro de Supercomputación de Galicia (CESGA)" for the use of their computational facilities. DFC thanks Xunta de Galicia for financial support through a postdoctoral grant.

\section{Authors' contributions}

AFR conceived and coded a preliminary version of the algorithm. DFC contributed to multiple aspects of the algorithm, as well as programmed and implemented the current version of Torsiflex. DFC and IML applied Torsiflex to the amino acids and performed the analysis of the results. DFC and AFR wrote the manuscript. All authors read and approved the final manuscript.

\section{Funding}

This work was partially supported by the Ministerio de Ciencia e Innovación (Grant \# PID2019-107307RB-I00), the Consellería de Cultura, Educación e Ordenación Universitaria (Centro singular de investigación de Galicia acreditación 2019-2022, ED431G 2019/03), and the European Regional Development Fund (ERDF)

\section{Availability of data and materials}

The manual of Torsiflex and the source code, are available at: https:// github.com/cathedralpkg/TorsiFlex The LL and HL Cartesian coordinates of the conformers, the reference Z-matrix and the Tors iflex input files for the 20 amino acids are available at: https://github.com/cathedralpkg/Aminoacids. 


\section{Declarations}

\section{Competing interests}

The authors declare that they have no competing interests.

Received: 15 April 2021 Accepted: 8 December 2021

Published online:: 24 December 2021

\section{References}

1. Metropolis N, Rosenbluth AW, Rosenbluth MN, Teller AH, Teller E (1953) Equation of state calculations by fast computing machines. J Chem Phys 21:1087-1092

2. Kirkpatrick S, Gelatt CD Jr, Vecchi MP (1983) Optimization by simulated annealing. Science 220:671-680

3. Li Z, Scheraga HA (1987) Monte Carlo-minimization approach to the multiple-minima problem in protein folding. Proc Nat Acad Sci USA 84:6611-6615

4. Cerjan CJ, Miller WH (1981) On finding transition states. J Chem Phys 75:2800-2806

5. Kolossváry I, Guida WC (1996) Low mode search. an efficient, automated computational method for conformational analysis: Application to cyclic and acyclic alkanes and cyclic peptides. J Am Chem Soc 118:5011-5019

6. Kamachi T, Yoshizawa K (2016) Low-mode conformational search method with semiempirical quantum mechanical calculations: application to enantioselective organocatalysis. J Chem Inf Model 56:347-353

7. Lipton M, Still WC (1988) The multiple minimum problem in molecular modeling. Tree searching inernal coordinate conformational space. J Comput Chem 9:343-355

8. Chan L, Hutchison GR, Morris GM (2019) Bayesian optimization for conformer generation. J Cheminformatics 11:1-11

9. Chan L, Hutchison GR, Morris GM (2020) BOKEl: Bayesian optimization using knowledge of correlated torsions and expected improvement for conformer generation. Phys Chem Chem Phys 22:5211-5219

10. Fang L, Makkonen E, Todorović M, Rinke P, Chen X (2021) Efficient amino acid conformer search with bayesian optimization. J Chem Theory Comput 17:1955-1966

11. Mancini G, Fusè M, Lazzari F, Chandramouli B, Barone V (2020) Unsupervised search of low-lying conformers with spectroscopic accuracy: a two-step algorithm rooted into the island model evolutionary algorithm. J Chem Phys 153:124110

12. Grimme S (2019) Exploration of chemical compound, conformer, and reaction space with meta-dynamics simulations based on tight-binding quantum chemical calculations. J Chem Theory Comput 15:2847-2862

13. Mohamadi F, Richards NGJ, Guida WC, Liskamp R, Lipton M, Caufield C, Chang G, Hedrickson T, Still WC (1990) Macromodel - an integrated software system for modeling organic and bioorganic molecules using molecular mechanics. J Comput Chem 11:440-467

14. Hawkins PCD, Skillman G, Warren GL, Ellingson BA, Stahl MT (2010) Conformer generation with OMEGA: Algorithm and validation using high quality structures from the protein databank and Cambridge structural database. J Chem Inf Model 50:572-584

15. Watts KS, Dalal P, Murphy RB, Sherman W, Friesner RA (2010) Confgen: a conformational search method for efficient generation of bioactive conformers. J Chem Inf Model 40:534-546

16. O'Boyle NM, Vandermeersch T, Flynn CJ, Maguire AR, Hutchison GR (2011) Confab - systematic generation of diverse low-energy conformers. Cheminformatics 3:8

17. Hawkins PCD, Nicholls A (2012) Conformer generation with OMEGA: learning from the data set and the analysis of failures. J Chem Inf Model 52:5219-5236

18. Hawkins PCD (2017) Conformation generation: the state of the art. J Chem Inf Model 57:1747-1756

19. Friedrich NO, Flachsenberg F, Meyder A, Sommer K, Kirchmair J, Rarey M (2019) Conformator: a novel method for the generation of conformer ensembles. J Chem Inf Model 59:731-742

20. Pracht P, Bohle F, Grimme S (2020) Automated exploration of the lowenergy chemical space with fast quantum chemical methods. Phys Chem Chem Phys 22:7169-7192
21. Chandramouli B, Del Galdo S, Fusè M, Barone V Mancini G (2019) Twolevel stochastic search of low-energy conformers for molecular spectroscopy: implementation and validation of MM and QM models. Phys Chem Chem Phys 21:19921-19934

22. Riniker S, Landrum GA (2015) Better informed distance geometry: using what we know to improve conformation generation. J Chem Inf Model 55:2562-2574

23. Ferro-Costas D, Fernández-Ramos A (2020) A combined systematicstochastic algorithm for the conformational search in flexible acyclic molecules. Front Chem 8:16

24. Smellie A, Kahn SD, Teig SL (1995) Analysis of conformational coverage. 1. Validation and estimation of coverage. J Chem Inf Model 35:285-294

25. Puzzarini C, Barone V (2020) The challenging playground of astrochemistry: an integrated rotational spectroscopy - quantum chemistry strategy. Phys Chem Chem Phys 22:6507-6523

26. Kolesniková L, León I, Alonso ER, Mata S, Alonso JL (2019) Laser ablation assists cyclation reactions of hydantoic acid: a proof for the near-attack conformation theory. J Phys Chem Lett 10:1325-1330

27. Ferro-Costas D, Martínez-Núñez E, Rodríguez-Otero J, Cabaleiro-Lago E, Estévez CM, Fernández B, Fernández-Ramos A, Vázquez SA (2018) Influence of multiple conformations and paths on rate constants and product branching ratios. thermal decomposition of 1-propanol radicals. J Phys Chem A 122:4790-4800

28. Zheng J, Yu T, Papajak E, Alecu IM, Mielke SL, Truhlar DG (2011) Practical methods for including torsional anharmonicity in thermochemical calculations on complex molecules: The internal-coordinate multi-structural approximation. Phys Chem Chem Phys 13:10885-10907

29. Meana-Pañeda R, Fernández-Ramos A (2012) Tunneling and conformational flexibility play critical roles in the isomerization mechanism of vitamin D. J Am Chem Soc 134:346-354

30. Fernández-Ramos A (2013) Accurate treatment of two-dimensional non-separable hindered internal rotors. J Chem Phys 138:134112

31. Simón-Carballido L, Bao JL, Alves TV, Meana-Pañeda R, Truhlar DG, Fernández-Ramos A (2017) Anharmonicity of coupled torsions: the extended two-dimensional torsion method and its use to assess more approximate methods. J Chem Theory Comput 13:3478-3492

32. Ferro-Costas D, Cordeiro MNDS, Truhlar DG, Fernández-Ramos A (2018) Q2DTor: a program to treat torsional anharmonicity through coupled pair of torsions in flexible molecules. Comput Phys Commun 232:190-205

33. Zheng J, Truhlar DG (2013) Quantum thermochemistry: multistructural method with torsional anharmonicity based on a coupled torsional potential. J Chem Theory Comput 9:1356-1367

34. Zheng J, Meana-Pañeda R, Truhlar DG (2013) MSTor version 2013: a new version of the computer code for the multi-structural torsional anharmonicity, now with a coupled torsional potential. Comput Phys Commun 184:2032-2033

35. Chan L, Morris GM, Hutchison GR (2021) Understanding conformational entropy in small molecules. J Chem Inf Model 17:2099-2106

36. Pracht P, Grimme S (2021) Calculation of absolute molecular entropies and heat capacities made simple. Chem Sci 12:6551-6568

37. Behr A, Seidensticker T (2020) Chemistry of Renewables. An Introduc tion, Springer, Berlin, Heidelberg, chap 14: Building Blocks of LifeAmino Acids, pp 251-264

38. Lesarri A, Sánchez R, Cocinero EJ, López JC, Alonso JL (2005) Coded amino acids in gas phase: the shape of isoleucine. J Am Chem Soc 127:12952-12956

39. Sanz AE, Blanco S, López JC, Alonso JL (2008) Rotational probes of six conformers of neutral cysteine. Angew Chem Int Ed 47:6216-6220

40. Alonso JL, Pérez C, Sanz ME, López JC, Blanco S (2009) Seven conformers of I-threonine in the gas phase: a LA-MB-FTMW study. Phys Chem Chem Phys 11:617-627

41. Mata S, Vaquero V, Cabezas C, Peña I, Pérez C, López JC, Alonso JL (2009) Observation of two new conformers of neutral proline. Phys Chem Chem Phys 11:4141-4144

42. Sanz ME, López JC, Alonso JL (2010) Six conformers of neutral aspartic acid identified in the gas phase. Phys Chem Chem Phys 12:3573-3578

43. Pérez C, Mata S, Blanco S, López JC, Alonso JL (2011) Jet-cooled rotational spectrum of laser-ablated phenylalanine. J Phys Chem A 115:9653-9657 
44. Peña I, Sanz ME, López JC, Alonso JL (2012) Preferred conformers of proteinogenic glutamic acid. J Am Chem Soc 134(4):2305-2312

45. Sanz ME, Cabezas C, Mata S, Alonso JL (2014) Rotational spectrum of tryptophan. J Chem Phys 140:8

46. Bermúdez C, Mata S, Cabezas C, Alonso JL (2014) Tautomerism in neutral histidine. Angew Chem Int 53:11015-11018

47. Pérez C, Mata S, Cabezas C, López JC, Alonso JL (2015) The rotational spectrum of tyrosine. J Phys Chem A 119:3731-3735

48. Grunenberg A, Bougeard D (1987) Vibrational spectra and conformational phase transition of crystalline I-methionine. J Mol Struct 160(1-2):27-36

49. Gronert S, O'Hair RA (1995) Ab initio studies of amino acid conformations. 1. the conformers of alanine, serine, and cysteine. J Am Chem Soc 117(7):2071-2081

50. Császár AG (1996) Conformers of gaseous $\alpha$-alanine. J Phys Chem 100:3541-3551

51. Sun W, Kinsel GR, Marynick DS (1999) Computational estimates of the gas-phase basicity and proton affinity of glutamic acid. J Phys Chem A 103(20):4113-4117

52. Czinki E, Császár AG (2003) Conformers of gaseous proline. Chem Eur J 9(4):1008-1019

53. Huang Z, Lin Z (2005) Detailed ab initio studies of the conformers and conformational distributions of gaseous tryptophan. J Phys Chem A 109(11):2656-2659

54. Zhang M, Huang Z, Lin Z (2005) Systematic ab initio studies of the conformers and conformational distribution of gas-phase tyrosine. J Chem Phys 122(13):134313

55. Chen M, Huang Z, Lin Z (2005) Ab initio studies of gas phase asparagine conformers. Theochem 719(1-3):153-158

56. Ling S, Yu W, Huang Z, Lin Z, Harañczyk M, Gutowski M (2006) Gaseous arginine conformers and their unique intramolecular interactions. J Phys Chem A 110(44):12282-12291

57. Huang Z, Yu W, Lin Z (2006) Exploration of the full conformational landscapes of gaseous aromatic amino acid phenylalanine: An ab initio study. Theochem 758(2-3):195-202

58. Chen M, Lin Z (2007) Ab initio studies of aspartic acid conformers in gas phase and in solution. J Chem Phys 127(15):154314

59. Schlund S, Müller R, Graßmann C, Engels B (2008) Conformational analysis of arginine in gas phase-a strategy for scanning the potential energy surface effectively. J Comput Chem 29(3):407-415

60. Balabin RM (2009) Conformational equilibrium in glycine: Focal-point analysis and ab initio limit. Chem Phys Lett 479(4-6):195-200

61. Leng Y, Zhang M, Song C, Chen M, Lin Z (2008) A semi-empirical and ab initio combined approach for the full conformational searches of gaseous lysine and lysine-h2o complex. Theochem 858(1-3):52-65

62. Wilke JJ, Lind MC, Schaefer HF III, Császár AG, Allen WD (2009) Conformers of gaseous cysteine. J Chem Theory Comput 5:1511-1523

63. Szidarovszky T, Czakó G, Császár AG (2009) Conformers of gaseous threonine. Mol Phys 107(8-12):761-775

64. Tehrani ZA, Tavasoli E, Fattahi A (2010) Conformational behavior and potential energy profile of gaseous histidine. Theochem 960(1-3):73-85

65. Dokmaisrijan S, Lee VS, Nimmanpipug P (2010) The gas phase conformers and vibrational spectra of valine, leucine and isoleucine: an AB initio study. Theochem 953(1-3):28-38

66. Riffet V, Frison G, Bouchoux G (2011) Acid-base thermochemistry of gaseous oxygen and sulfur substituted amino acids (Ser, Thr, Cys, Met). Phys Chem Chem Phys 13:18561-18580

67. Rode JE, Dobrowolski JC, Sadlej J (2013) Prediction of I-methionine VCD spectra in the gas phase and water solution. J Phys Chem A 117(46):14202-14214

68. Pang R, Guo M, Ling S, Lin Z (2013) Thorough theoretical search of conformations of neutral, protonated and deprotonated glutamine in gas phase. Comput Theor Chem 1020:14-21

69. Turan B, Selçuki C (2014) Conformational analysis of glutamic acid: a density functional approach using implicit continuum solvent model. J Mol Model 20(9):2396

70. Jamróz MH, Rode JE, Ostrowski S, Lipiński PFJ, Dobrowolski JC (2012) Chirality measures of $\alpha$-amino acids. J Chem Inf Model 52(6):1462-1479

71. Ropo M, Schneider M, Baldauf C, Blum V (2016) First-principles data set of 45,892 isolated and cation-coordinated conformers of 20 proteinogenic amino acids. Sci Data 3(1):1-13
72. Ropo M, Blum V Baldauf C (2016) Trends for isolated amino acids and dipeptides: conformation, divalent ion binding, and remarkable similarity of binding to calcium and lead. Sci Rep 6(1):1-11

73. Perdew JP, Burke K, Ernzerhof M (1996) Generalized gradient approximation made simple. Phys Rev Lett 77:3865

74. Frisch MJ, Trucks GW, Schlegel HB, Scuseria GE, Robb MA, Cheeseman JR, Scalmani G, Barone V, Petersson GA, Nakatsuji H, Li X, Caricato M, Marenich A, Bloino J, Janesko BG, Gomperts R, Mennucci B, Hratchian HP, Ortiz JV, Izmaylov AF, Sonnenberg JL, Williams-Young D, Ding F, Lipparini F, Egidi F, Goings J, Peng B, Petrone A, Henderson T, Ranasinghe D, Zakrzewski VG, Gao J, Rega N, Zheng G, Liang W, Hada M, Ehara M, Toyota K, Fukuda R, Hasegawa J, Ishida M, Nakajima T, Honda Y, Kitao O, Nakai H, Vreven T, Throssell K, J A Montgomery J, Peralta JE, Ogliaro F, Bearpark M, Heyd JJ, Brothers E, Kudin KN, Staroverov VN, Keith T, Kobayashi R, Normand J, Raghavachari K, Rendell A, Burant JC, lyengar SS, Tomasi J, Cossi M, Millam JM, Klene M, Adamo C, Cammi R, Ochterski JW, Martin RL, Morokuma K, Farkas O, Foresman JB, Fox DJ (Gaussian 09, Revision A.02; Gaussian, Inc., Wallingford, CT, 2004)

75. Frisch MJ, Trucks GW, Schlegel HB, Scuseria GE, Robb MA, Cheeseman JR, Scalmani G, Barone V, Petersson GA, Nakatsuji H, Li X, Caricato M, Marenich AV, Bloino J, Janesko BG, Gomperts R, Mennucci B, Hratchian HP, Ortiz JV, Izmaylov AF, Sonnenberg JL, Williams-Young D, Ding F, Lipparini F, Egidi F, Goings J, Peng B, Petrone A, Henderson T, Ranasinghe D, Zakrzewski VG, Gao J, Rega N, Zheng G, Liang W, Hada M, Ehara M, Toyota K, Fukuda R, Hasegawa J, Ishida M, Nakajima T, Honda Y, Kitao O, Nakai H, Vreven T, Throssell K, Montgomery JA Jr, Peralta JE, Ogliaro F, Bearpark MJ, Heyd JJ, Brothers EN, Kudin KN, Staroverov VN, Keith TA, Kobayashi R, Normand J, Raghavachari K, Rendell AP, Burant JC, lyengar SS, Tomasi J, Cossi M, Millam JM, Klene M, Adamo C, Cammi R, Ochterski JW, Martin RL, Morokuma K, Farkas O, Foresman JB, Fox DJ (2016) Gaussian 16 Revision B.01. Gaussian Inc. Wallingford CT

76. Binkley JS, Pople JA, Hehre WJ (1980) Self-consistent molecular orbital methods. 21. Small split-valence basis sets for first-row elements. J Am Chem Soc 102:939-947

77. Gordon MS, Binkley JS, Pople JA, Pietro WJ, Hehre WJ (1982) Self-consistent molecular orbital methods. 22. Small split-valence basis sets for second-row elements. J Am Chem Soc 104:2797-2803

78. Ríos MA, Rodríguez J (1992) Semiempirical study of compounds with O-H...O intramolecular hydrogen bond. J Comput Chem 13:860-866

79. Stewart JJP (2013) Optimization of parameters for semiempirical methods VI: more modifications to the NDDO approximations and re-optimization of parameters. J Mol Model 19:1-32

80. Bannwarth C, Caldeweyher E, Ehlert S, Hansen A, Pracht P, Seibert J, Spicher S, Grimme S (2020) Extended tight-binding quantum chemistry methods. WIREs Comput Mol Sci. https://doi.org/10.1002/wcms.1493

81. Zhao Y, Truhlar DG (2008) Exploring the limit of accuracy of the global hybrid meta density functional for main-group thermochemistry, kinetics, and noncovalent interactions. J Chem Theory Comput 4:1849-1868

82. Lynch BJ, Zhao Y, Truhlar D (2003) Effectiveness of diffuse basis functions for calculating relative energies by density functional theory. J Phys Chem A 107(9):1384

83. Fast PL, Sánchez ML, Truhlar DG (1999) Multi-coefficient gaussian-3 method for calculating potential energy surfaces. Chem Phys Lett 306:407-410

84. Mardirossian N, Head-Gordon M (2016) How accurate are the Minnesota density functionals for noncovalent interactions, isomerization energies, thermochemistry, and barrier heights involving molecules compose of main-group elements? J Chem Theory Comput 12:4303-4325

85. Bao JL, Sripa P, Truhlar DG (2016) Path-dependent variational effects and multidimensional tunneling in multi-path variational transition state theory: rate constants calculated for the reactions of $\mathrm{ho}_{2}$ with tert-butanol by including all 46 paths for abstraction at $C$ and all six paths for abstraction at O. Phys Chem Chem Phys 18:1032-1041

86. Gao L, Zheng J, Fernández-Ramos A, Truhlar DG, Xu X (2018) Kinetics of the methanol reaction with $\mathrm{OH}$ at interstellar, atmospheric, and combustion temperatures. J Am Chem Soc 140:2906-2918

87. Alecu IM, Zheng J, Zhao Y, Truhlar DG (2010) Computational thermochemistry: scale factor databases and scale factors for vibrational frequencies obtained from electronic model chemistries. J Chem Theory Comput 6:2872-2887 
88. Ferro-Costas D, Fernández-Ramos A (2021) The cathedral package. https://github.com/cathedralpkg. Accessed 18 Dec 2021

89. Moss GP (1996) Basic terminology of stereochemistry (IUPAC recommendations 1996). Pure Appl Chem 68:2193-2222

90. IUPAC \& IUB (1984) Nomenclature and symbolism for amino acids and peptides. Pure Appl Chem 56:595-624

91. Stewart JJP (2007) Optimization of parameters for semiempirical methods V: modifications of NDDO and application to 70 elements. J Mol Model 13:1173-1213

92. Landrum G (2021) RDKit: Open-source cheminformatics and machine learning. http://www.rdkit.org

93. Yirik MA, Sorokina M, Steinbeck C (2021) MAYGEN: an open-source chemical structure generator for constitutional isomers based on the orderly generation principle. J Cheminformatics 13:48

\section{Publisher's Note}

Springer Nature remains neutral with regard to jurisdictional claims in published maps and institutional affiliations.

- fast, convenient online submission

- thorough peer review by experienced researchers in your field

- rapid publication on acceptance

- support for research data, including large and complex data types

- gold Open Access which fosters wider collaboration and increased citations

- maximum visibility for your research: over $100 \mathrm{M}$ website views per year

At BMC, research is always in progress.

Learn more biomedcentral.com/submissions 